quartiers à " taux cellulaires élevés ", le nombre des leucocytes n'est jamais descendu au-dessous de 1.000 .000 par centimètre cube. En d'autres termes, dès qu'un grand nombre de leucocytes est apparu dans le lait d'un quartier quelconque, ce nombre diminue rarement.

Les mêmes observations furent trouvées exactes en ce qui concerne le lait contenant un petit nombre de leucocytes. Le nombre maximum trouvé dans le lait provenant de quartiers ne présentant pas de tissu cicatriciel et étudiés pendant une période de dix mois, n'excéda jamais 200.000 par centimètre cube, les animaux restant, pendant la période d'essai entière, des vaches à " taux cellulaire bas 》.

On nota une certaine proportion de variation entre la teneur en leucocytes du lait des quatre quartiers de la même bête. Une des observations les plus intéressantes faite à cet égard fut qu'on trouva une plus grande variation dans le lait provenant de quartiers ayant un nombre excessif de leucocytes que dans le lait en contenant un nombre minimum.

(A suivre.)

\title{
ÉTUdE DE L'APPRÉCIATION DE LA QUALITÉ DES CASÉINES LACTIQUES
}

\author{
par \\ JEAN PIEN \\ Ingénieur chimiste I. C. R. \\ Docteur ès sciences \\ Directeur des laboratoires \\ des a Fermiers Réunis "

\section{MARO BERGIER} \\ Ingénieur (I. A. N.) \\ du laboratoire des caséines \\ des " Fermiers Réunis "
}

(Suite.)

Nous croyons avoir montré dans notre précédent article pourquoi l'analyse chimique ordinaire des caséines lactiques doit être considérée comme insuffisante à déceler certains défauts de fabrication. Nous avons proposé de faire appel à une méthode d'examen complémentaire, dont nous devons rappeler, en quelques mots, le principe.

L'examen systématique de chaque point de la fabrication de la caséine lactique conduit, selon nous, à admettre comme bases de travail les trois conditions suivantes:

$1^{\circ} \mathrm{Ne}$ produire aucune protéine dégradée à partir de la caséine initiale.

$2^{\circ}$ Ne fixer sur la molécule protéique aucun élément qui n'y soit lié naturellement.

$3^{\circ}$ Ne laisser en mélange avec la caséine produite aucune substance organique ou minérale. 
En dernière analyse toute la fabrication de la caséine lactique tient dans ces trois termes. Nous avons montré dans un article antérieur (Le Lait, janvier 1934) comment ces conditions peuvent être réalisées dans la pratique industrielle.

Notre but est maintenant de montrer par quels procédés d'examen chimique on peut découvrir qu'une caséine a été fabriquée dans de mauvaises conditions, c'est-à-dire en négligeant l'un ou l'autre de ces principes essentiels.

La méthode que nous proposerons se ramène à la prise en considération simultanée des résultats de l'analyse chimique ordinaire, et de ceux de l'analyse des macérations aqueuses et des solutions de caséine. Les éléments qui doivent faire l'objet de ces analyses sont: l'azote soluble sous ses différentes formes, l'acide lactique, le lactose. les matières minérales (calcium et phosphore principalement). Le présent article traite du dosage des différentes formes d'azote soluble dans les caséines lactiques.

$$
*^{*} *
$$

\section{§1. L'ORIGINE DE L'AZOTE SOLUBLE DANS LES CASEINES INDUSTRIELLES}

La constitution chimique des protéines a fait l'objet de nombreuses théories, dont les plus récentes et les plus communément admises actuellement, reposent sur les travaux de FIScHER et surtout d'ABDERHALDEN.

On admet que les protéines sont formées d'assemblages complexes de cyclopeptides, de peptides et d'amino-acides. Sur la nature de la liaison de ces divers éléments entre eux on n'est pas encore d'accord. Des hypothèses parfaitement contradictoires (Pfeiffer, Hess, Pringsheim) ont été échafaudées. Mais on est à peu près certain maintenant que les protéines ne sont pas exclusivement formées de polypeptides, ni de noyaux cyclopeptiques simples, mais de cycles à chaînes latérales.

Cette structure de la molécule de la protéine (valable pour la caséine) permet de prévoir la libération possible sous l'influence de certaines causes, de chaînes polypeptidiques simples, voire même d'acides aminés. On conçoit donc que, sous l'influence d'une hydrolyse par exemple, la caséine, qui est une protéine insoluble dans l'eau, libère des formes d'azote soluble.

Une fabrication normale, typique, doit toujours conduire à la caséine pure, théorique, insoluble dans l'eau. Quelles sont, au cours d'une fabrication industrielle, les causes susceptibles de produire de l'azote soluble?

10 Le lait contient normalement des formes d'azote soluble. 
S'il a été chauffé à une température suffisante, à côté de l'insolubilisation de certaines protéines (albumines) on voit par contre se produire une augmentation du taux de ses acides aminés. Des travaux récents ont montré que cette teneur pouvait doubler. Ce sera le cas d'un lait pasteurisé écrémé, destiné ensuite à la fabrication de la caséine. De toute façon, cru ou pasteurisé, le lait apporte avec lui de l'azote soluble, non précipitable pendant la fermentation lactique et dont on pourra ensuite se débarrasser par de sérieux lavages.

$2^{\circ} \mathrm{La}$ fermentation lactique ne peut guère être le siège d'une solubilisation de l'azote protéinique du moins dans les conditions de la fabrication de la caillebotte; car l'acidité produite s'oppose en général à l'action de beaucoup de microbes hydrolysants. Il faut noter toutefois que l'action des acido-protéolytiques n'est nullement incompatible avec celle des ferments lactiques et qu'elle peut jouer un rôle même pendant la durée relativement courte de la fermentation lactique. Ces formes d'azote sont également appelées à disparaître au cours du lavage.

$3^{\circ}$ La caillebotte lavée, pressée, si elle n'est pas immédiatement séchée peut subir (en été surtout) des fermentations très rapidement hydrolysantes, de véritables commencements de putréfaction. Ce phénomène est d'autant plus à redouter qu'on aura mieux lavé la caillebotte et qu'on l'aura ainsi mieux débarrassée de l'acide lactique où elle a pris naissance. Mais l'action antiseptique de l'acide lactique est limitée et il est courant de voir des caillebottes mal lavées, ou non lavées, subir un commencement de putréfaction, moins intense peut-être, mais nullement négligeable. Comme, pour d'autres raisons, on est tenu de laver à fond la caillebotte avant de la sécher, il s'ensuit que l'hydrolyse fermentaire est à redouter à chaque fois que le séchage ne $s^{\prime}$ effectue pas immédiatement après le pressage. (Notons en passant qu'il existe des moyens d'éviter cet inconvénient, mais nous n'avons pas à les envisager ici.)

Donc, en général, on peut craindre la libération des formes d'azote soluble dans la caillebotte - formes d'azote qui ne seront plus enlevées puisque le produit ne sera plus lavé - et qui sécheront avec la caillebotte. Si l'altération est faible, l'aspect de la caséine ne sera pas modifié, mais ses propriétés seront altérées et l'analyse habituelle n'aura pas pu le prévoir parce que le taux d'azote total n'aura pratiquement pas varié.

De ces trois facteurs de présence d'azote soluble, c'est évidemment le dernier qui est le plus important, puisque les deux premiers ont des chances d'être compensés par le lavage. C'est aussi, de beaucoup, celui qui peut devenir le plus intense. Comme il passe 
presque toujours inaperçu dans la caséine fabriquée, c'est là un inconvénient très sérieux.

Nous avons d'ailleurs acquis la conviction que de nombreux accidents de fabrication dans l'utilisation de la caséine pouvaient être rapportés à la présence de formes d'azote soluble. C'est assez dire l'intérêt que nous attachons à la détermination de cet élément.

\section{§ 2. LA TEOHNIQUE dU DOSAGE DE L'AZOTE SOLUBLE GLOBAL}

I. Principe. - Il est facile de concevoir une technique de ce dosage. Une caséine finement broyée mise en suspension dans l'eau doit abandonner progressivement tout ce qu'elle contenait de soluble.

Mais deux questions se posent :

10 La dissolution n'est certainement pas instantanée. Combien de temps faut-il maintenir la caséine en suspension dans l'eau ?

$2^{\circ}$ Si le contact doit se prolonger, ne risque-t-on pas de voir se produire une hydrolyse partielle de la caséine, soit sous l'influence des microbes qui la souillent inévitablement, soit même simplement par voie d'hydrolyse chimique?

II. II y a hydrolyse de la caséine dans l'eau. - 50 gr. de caséine lactique (fabriquée dans d'excellentes conditions, lavée 5 fois, séchée aussitôt et finement moulue) sont mis à macérer à la température ordinaire dans un litre d'eau distillée. Au bout d'une heure, 24, 48, 70 heures de contact entrecoupé d'agitations fréquentes, on centrifuge, dans les conditions données plus loin, une partie de l'eau décantée et on prélève $60 \mathrm{~cm}^{3}$ (= $3 \mathrm{gr}$. de caséine) du filtrat.

On concentre en présence d'acide sulfurique et on dose l'azote contenu dans ce filtrat par la méthode kjeldahl. Pour faciliter la comparaison des résultats nous avons multiplié les taux d'azote par le coefficient 6,4, comme s'il s'agissait d'azote de la caséine. Cette petite inexactitude ne modifie pas la conclusion. Nous avons obtenu :

\begin{tabular}{|c|c|c|}
\hline & Azote solul & le la caséine \\
\hline 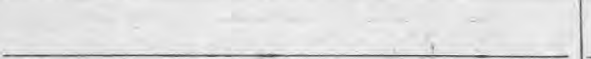 & En azote & Eln caséine \\
\hline 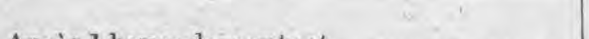 & & \\
\hline Après 1 heure de contact ............. & 0,18 & 1,15 \\
\hline Après 24 heures de contact............. & 0,33 & 2,11 \\
\hline Après 48 heures de contact. . . . . . . . . . . & 0,43 & 2,75 \\
\hline Après 70 heures de contact. . . . . . . . . . & 0,54 & 3,45 \\
\hline
\end{tabular}

Il y a donc une augmentation continue du taux d'azote soluble.

Certains essais nous ont donné des hydrolyses plus élevées encore.

On ne peut pas invoquer la lenteur de la dissolution des formes d'azote soluble originelles puisque nous nous sommes mis dans la 
situation de n'en pas avoir. Il faut admettre qu'il y a une hydrolyse véritable.

III. L'hydrolyse est d'origine fermentaire et est empêchée par le chloroforme. - Il s'agissait de savoir si l'hydrolyse pouvait être empêchée.

Si elle est d'origine bactérienne on doit l'atténuer ou l'empêcher avec le chloroforme. Si elle est d'origine chimique, elle doit persister en présence de chloroforme.

Les mêmes essais ont été répétés en introduisant dans l'eau un léger excès de chloroforme (10 $\mathrm{cm}^{3}$ pour 1 litre). Résultats obtenus :

Après 8 heures de contact

Après 24 heures de contact.

Après 48 heures de contact

Après 72 heures de contact............

\begin{tabular}{|c|c|}
\hline \multicolumn{2}{|c|}{ Azote solubilisé \% de caséine } \\
\hline En azote & En caséine \\
\hline 0,15 & 0,96 \\
0,18 & 1,15 \\
0,18 & 1,15 \\
0,19 & 1,21 \\
\hline
\end{tabular}

Plusieurs essais effectués dans les mêmes conditions (voir ciaprès) ont confirmé ce résultat que l'hydrolyse observée est d'origine fermentaire, puisqu'on peut la supprimer par le chloroforme.

IV. II n'y a pas d'hydrolyse chimique même après de très longs contacts. - Les mêmes essais, avec chloroforme, ont été effectués sur diverses caséines, toujours préparées, lavées et séchées avec soin. Les essais ont été prolongés pendant 9 jours.

A titre de curiosité, nous avons mené parallèlement des essais de macération sur la caséine, et aussi sur la caillebotte non séchée dont elle provenait - de manière à échapper à l'objection éventuelle d'une plus grande difficulté de réaction entre la caséine séchée et l'eau.

Voici un exemple des résultats obtenus :

\begin{tabular}{|c|c|c|}
\hline$=7$ & \multicolumn{2}{|c|}{ Azote solubilisé \% de caséine sèche } \\
\hline & $\begin{array}{c}\text { Essai sur } \\
\text { la vaillebotte }\end{array}$ & $\begin{array}{c}\text { Essai sur la } \\
\text { casóine }\end{array}$ \\
\hline Après 2 heures de contact. . . . . . . . . . & 0,14 & 0,10 \\
\hline Après 5 heures de contact............ & 0,14 & 0,13 \\
\hline Après 8 heures de contact.............. & 0,16 & 0,13 \\
\hline Après 24 heures de contact $\quad \ldots \ldots \ldots \ldots$ & 0,16 & 0,15 \\
\hline Après 72 heures de contact $\ldots$ & 0,17 & 0,18 \\
\hline Après 110 heures de contact............ & 0,18 & 0,21 \\
\hline Après 168 heures de contact. . . . . . . . . . & 0,18 & 0,22 \\
\hline Après 216 heures de contact........... & 0,18 & 0,22 \\
\hline
\end{tabular}


Ces chiffres sont comparables entre eux et confirment l'essai précédent. Ils montrent qu'on n'a pas l'hydrolyse chimique. (On observe une certaine période d'accroissement de l'azote au début, qui correspond vraisemblablement à la mise en solution de traces d'azote soluble pratiquement inévitables.)

Par conséquent nous pouvons estimer que la méthode qui consistera à mettre caillebotte ou caséine en macération dans l'eau chloroformée, nous permettra de dissoudre l'azote soluble sans introduire une cause d'erreur qui serait la solubilisation progressive de la caséine non hydrolysée. Il va maintenant nous être facile de déterminer quelle devra être la durée de macération pour obtenir la dissolution complète de tout l'azote soluble.

V. Durée de la macération. - Les chiffres obtenus précédemment nous éclairent déjà un peu à cet égard. Mais l'expérience devait être tentée, bien entendu, avec des produits contenant certainement de l'azote soluble.

Nous avons estimé que la cause essentielle de la présence de l'azote soluble dans une caséine était la fermentation de la caillebotte entre le pressage et le séchage. Nous avons done réalisé de tels produits en abandonnant à une température d'environ $30^{\circ}$ pendant des temps variables des caillebottes bien lavées et pressées. Nous avons également réalisé des produits où les autres formes d'azote soluble existaient seules (azote soluble du lait ou produit éventuellement par la fermentation lactique) en ne lavant pas certaines caillebottes qui d'ailleurs étaient séchées immédiatement pour éliminer toute putréfaction.

Premier exemple : Caséine provenant d'une caillebotte lavée puis fermentée fortement.

Mêrne technique de macération que dans les cass précédents. Résultats obtenus :

\begin{tabular}{|c|c|c|}
\hline - & \multicolumn{2}{|c|}{ Azote solubilisé \% de caséine } \\
\hline$=$ & En azote & En caséine \\
\hline Après 2 heures de macération ........... & 7 & 44,8 \\
\hline Après 5 heures de macération . . . . . . . . . . & 7,26 & 46,5 \\
\hline Après 8 heures de macération .......... & 7,41 & 47,4 \\
\hline Après 24 heures de macération .......... & 7,6 & 48,6 \\
\hline Après 48 heures de macération .......... & 7,7 & 49,2 \\
\hline
\end{tabular}

Ces chiffres et surtout la courbe qu'ils ont permis de tracer montrent que la dissolution paraît achevée (ou tout près de l'être) en 48 heures de macération, la majeure partie de l'azote étant déjà dissous en quelques heures. 
Deuxième exemple: Caséine provenant d'une caillebotte non lavée mais non fermentée.

Nous avons, en même temps, réalisé l'essai sur la caséine provenant de la même caillebotte lavée à fond (et non fermentée).

Voici les résultats exprimés en azote :

\begin{tabular}{|c|c|c|}
\hline 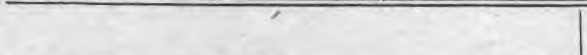 & Azote solubili & sé \% de caséine \\
\hline$=0$ & Caillebotte lavée & Caillebotte non lavée \\
\hline Après 2 heures de macération ........... & 0,19 & 0,25 \\
\hline Après 18 heures de macération ......... & 0,24 & 0,38 \\
\hline Après 48 heures de macération .......... & 0,32 & 0,43 \\
\hline Après 72 heures de macération ......... & 0,32 & 0,46 \\
\hline Après 96 heures de macération $\ldots \ldots \ldots \ldots$ & 0,32 & 0,46 \\
\hline
\end{tabular}

Nous avons atteint le maximum de la dissolution de l'azote soluble en 48-72 heures. Il n'y a d'ailleurs aucun inconvénient à prolonger la macération plus longtemps.

Notons en passant ce fait curieux qu'une caséine provenant d'une caillebotte non lavée contient à peine plus d'azote soluble que la caséine provenant de la même caillebotte lavée 5 fois. Il faut admettre que l'azote soluble apporté par le lait est très faible. Il représente environ $1 \%$ de l'azote total de la caséine. Il ne faudrait naturellement pas en conclure que le lavage de la caillebotte est une opération inutile ! La teneur en matières minérales de la caséine en dépend beaucoup.

Voici un autre essai analogue confirmant les précédents, effectué sur une caséine provenant également de caillebotte non lavée, non fermentée. L'essai a été effectué à la fois sur la caillebotte et sur la caséine :

\begin{tabular}{|c|c|c|}
\hline & Azote solutilisé & \% de caséine \\
\hline+ & Essai sur caillebotte & Essai sur caséine : \\
\hline Après 2 heures en contact............. & 0,28 & 0,33 \\
\hline Après 5 heures en contact. . . . . . . . . . & 0,30 & 0,35 \\
\hline Après 8 heures en contact. . . . . . . . . . & 0,30 & 0,36 \\
\hline Après 24 heures en contact. . . . . . . . . . & 0,31 & 0,38 \\
\hline Après 48 heures en contaet . . . . . . . . . . & 0,33 & 0,38 \\
\hline Après 72 heures en contact........... & 0,35 & 0,39 \\
\hline Après 120 heures en contact. . . . . . . . . & 0,38 & 0,41 \\
\hline Après 168 heures en còntact . . . . . . . . . . & 0,38 & 0,43 \\
\hline Après 240 heures en contact............ & 0,38 & 0,43 \\
\hline
\end{tabular}


Ici, il nous a fallu prolonger la macération de 120 à 160 heures pour avoir des chiffres constants, mais il faut noter que plus des 9/10 de l'azote soluble sont extraits en 72 heures.

Comme il n'y a aucun inconvénient à prolonger la durée de contact il nous sera très facile de proposer une macération convenable.

VI. Technique proposée pour le dosage de l'azote soluble total d'une caséine. - On introduit dans un flacon quelconque d'un demi-litre environ : $200 \mathrm{~cm}^{3}$ d'eau distillée et $10 \mathrm{gr}$. de caséine finement broyée (tamis 90). On ajoute $2 \mathrm{~cm}^{3}$ de chloroforme. On bouche au caoutchouc et on laisse en contact 3 jours au moins en agitant plusieurs fois par jour. Il n'y a aucun inconvénient à laisser la macération se poursuivre plus longtemps, voire pendant une semaine. La macération est conduite à la température ordinaire de 15-20.

On prélève $120 \mathrm{~cm}^{3}$ de la macération et on centrifuge à 1.200 tours pendant 30 minutes. (On répartit ces $120 \mathrm{~cm}^{3}$ dans quelques tubes à essais de gros diamètre bouchés au caoutchouc. La centrifugation peut s'effectuer dans la centrifugeuse Gerber utilisée dans l'analyse du lait. Il suffit de caler les tubes à essais avec du coton dans les étuis en laiton des butyromètres.) Après la centrifugation toutes les matières solides qui étaient en suspension ultra-fine sont agglomérées. On décante sur un filtre pour retenir des conglomérats qui auraient pu se décoller.

On prélève $100 \mathrm{~cm}^{3}$ de ce filtrat que l'on concentre dans un ballon kjeldahl en présence de $5 \mathrm{~cm}^{3}$ d'acide sulfurique concentré. Quand le volume du liquide est ramené à 20-30 $\mathrm{cm}^{3}$ on ajoute après refroidissement, $20 \mathrm{~cm}^{3}$ d'acide sulfurique concentré, $10 \mathrm{gr}$. de sulfate de potassium, $1 \mathrm{gr} .5$ de sulfate de cuivre cristallisé et on procède à l'attaque selon Kjeldahl.

On distille ensuite dans les conditions habituelles (sans mettre de sulfure de sodium) et on recueille le distillat dans de l'acide N/10 ou N/1 suivant les cas. Le titrage en retour et un calcul simple permettent de déduire la dose d'azote libéré par la caséine pendant la macération aqueuse.

VII, Interprétation des résultats. - Nous reviendrons ultérieurement sur la mise en application de ces techniques. Nous pouvons néanmoins dire dès maintenant :

$1^{\circ}$ Qu'une caséine provenant d'une caillebotte très bien lavée (et non fermentée) abandonne par macération prolongée dans l'eau, une dose d'azote de l'ordre de $0,2 \%$ de la caséine initiale, c'est-à-dire environ 1,5\% de l'azote total.

$2^{\circ}$ Qu'une caséine provenant d'une eaillebotte non lavée (mais n'ayant pas fermenté par la suite) abandonne dans les 
mêmes conditions une dose d'azote de l'ordre de $0,3-0,4 \%$ de la caséine initiale, c'est-à-dire environ 2 à $3 \%$ de l'azote total.

$3^{\circ} \mathrm{Qu}$ 'une caséine provenant d'une caillebotte, lavée ou non, mais ayant fermenté entre le pressage et le séchage peut abandonner dans les mêmes conditions des doses d'azote variant de 0,2 à 6 et même $8 \%$ de la caséine initiale, c'est-à-dire de 1 à 50 et même $60 \%$ de son azote total, alors que la valeur absolue de cet azote total n'aura que très faiblement varié et ne permettra pas de soupçonner l'accident de fabrication qui s'est produit.

(A suivre.)

\section{CONTRIBUTION A L'ÉTUDE DES BEURRES BELGES (1)}

\section{par C. HUYGE}

Professeur à l'Institut agronomique, Directeur de la Station laitière de l'Etat à Gembloux.

\section{INTRODUCTION.}

Sous cette rubrique, la Station laitière se propose de publier plusieurs documents se rapportant aux beurres produits dans les laiteries belges. La Station laitière est en possession d'un nombre assez considérable de résultats analytiques fournis depuis dix ans par l'examen de plus de 20.000 échantillons de beurre prélevés, à raison d'un échantillon par quinzaine et par laiterie, dans 80 exploitations industrielles.

La fabrication fermière du beurre, encore très répandue en Belgique, mais destinée à disparaître peu à peu, n'a aucune place dans cette publication.

Les beurres examinés proviennent de laiteries plus ou moins importantes, depuis les exploitations modestes, si nombreuses dans le pays et produisant moins de $50.000 \mathrm{~kg}$. de beurre par an, jusqu'aux grosses laiteries du pays wallon, dont quelques-unes ont une fabrication annuelle dépassant $400.000 \mathrm{~kg}$. de beurre.

Tous les échantillons examinés ont été prélevés par les agents du Contrôle des Beurres Belges, sur la fabrication du jour et analysés par la Station laitière dans les trois jours.

Grâce à ce système nous avons pu réunir une documentation considérable et de premier ordre, se rapportant à des beurres frais, vierges de toute manipulation, chaque échantillon donnant la composition moyenne de la matière grasse produite par les vaches du rayon d'approvisionnement de chaque laiterie.

Les analyses ont porté et continuent à porter sur l'indice

(1) Paru dans le Bulletin de l'Institut Agronomique et des stations de Recherches de Gem. bloux, t. I, n० 4, novembre 1932. 